\title{
Control of multilayer biological networks and applied to target identification of complex diseases
}

\author{
Wei Zheng, Dingjie Wang and Xiufen Zou*
}

\begin{abstract}
Background: Networks have been widely used to model the structures of various biological systems. The ultimate aim of research on biological networks is to steer biological system structures to desired states by manipulating signals. Despite great advances in the linear control of single-layer networks, it has been observed that many complex biological systems have a multilayer networked structure and extremely complicated nonlinear processes.

Result: In this study, we propose a general framework for controlling nonlinear dynamical systems with multilayer networked structures by formulating the problem as a minimum union optimization problem. In particular, we offer a novel approach for identifying the minimal driver nodes that can steer a multilayered nonlinear dynamical system toward any desired dynamical attractor. Three disease-related biology multilayer networks are used to demonstrate the effectiveness of our approaches. Moreover, in the set of minimum driver nodes identified by the algorithm we proposed, we confirmed that some nodes can act as drug targets in the biological experiments. Other nodes have not been reported as drug targets; however, they are also involved in important biological processes from existing literature.
\end{abstract}

Conclusions: The proposed method could be a promising tool for determining higher drug target enrichment or more meaningful steering nodes for studying complex diseases.

Keywords: Multilayer networks, Network control, Nonlinear dynamical systems, IISG algorithm, Driver nodes

\section{Background}

Biological processes, which are indispensable for living organisms, are often carried out by complex interactions among various biological elements. Studying biological elements and their interactions is vital for understanding the roles of intracellular biomolecules and the mechanisms of biological processes. The structure of biological systems can be modeled by biological networks in which nodes are biological elements and edges connect biological elements that interact with one another. Therefore, networks have been widely used to model the structures of various biological systems. Moreover, the ultimate aim of research on biological networks is to steer the states of biological systems to desired states by manipulating signals. Recently, controllability, which is a

\footnotetext{
* Correspondence: xfzou@whu.edu.cn

School of Mathematics and Statistics, Wuhan University, Wuhan 430072 China
}

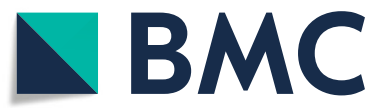

(c) The Author(s). 2019 Open Access This article is distributed under the terms of the Creative Commons Attribution 4.0 International License (http://creativecommons.org/licenses/by/4.0/), which permits unrestricted use, distribution, and

reproduction in any medium, provided you give appropriate credit to the original author(s) and the source, provide a link to the Creative Commons license, and indicate if changes were made. The Creative Commons Public Domain Dedication waiver (http://creativecommons.org/publicdomain/zero/1.0/) applies to the data made available in this article, unless otherwise stated. gate the dynamics of complex networks.

In the past few decades, the controllability of singlelayer networks with linear [1-5] and nonlinear [6-9] dynamics have been widely studied in a variety of biomedical systems. In particular, an innovative study of the newly developed linear control approaches is a structural controllability framework [2], that involves using a graph-based tool to identify the minimal driver nodes set by formulating this problem as a maximum matching problem. Then, many other methods related to linear control of single-layer networks were proposed $[1,5]$ and some important topics in network controllability, including observability [10], control energy [3, 11, 12], target control [13], etc., were discussed. With a deeper realization of network controllability, many researchers have observed that multiple real-world complex systems are extremely complicated nonlinear processes. Therefore, several new approaches have been proposed for 
controlling nonlinear single-layer networks [6-9]. In particular, a breakthrough in recently developed methods led to mapping the controllability of a single network to the feedback vertex set (FVS) problem in which the objective is to drive the nonlinear single networked system from an arbitrary initial state to any desired dynamical attractor (e.g., a steady state) by overriding the state of a certain node $[8,14]$. In our recent study $[15,16]$, we established a new theory of domain controllability to describe the controllability of nonlinear dynamical networks and demonstrated how to drive a complex networked system in transition from the attraction domain of a stable steady state to the attraction domain of another stable steady state.

However, with the development of network controllability theory, it has been shown that biological networks are always governed by multiple types of interactions or interact with other networks. Single networks might be insufficient to discover the underlying biological mechanism. For example, cellular activities in biochemical networks, gene regulatory networks, and a biochemical reactions network are all highly interdependent and are excellent choices for being analyzed as multilayer networks [17-19]. In recent years, a multilayer network method has been proposed to describe such complex, multidimensional biological systems. A multilayer network can link human diseases with genetic, biochemical, and environmental factors [20-23].

More recently, some advances [24-27] have focused on the linear control of multilayer networks. Menichetti et al. [25] proposed a combinatorial matching model to identify the minimal set of driver nodes in multilayer networks. Pósfai et al. [26] developed a theory based on disjoint path covers to determine the minimum number of inputs necessary for full control of multiplex, multiscale networks. Recent efforts have dedicated to understanding the interplay between the degree correlation of interconnections and the controllability of multilayer networks [27, 28].

However, from the perspective of a dynamical process, many biological systems are extremely complicated nonlinear dynamical processes such as cell signaling, information transmission $[8,29,30]$. Exploring the controllability properties of these real-world complex systems has fundamental importance and multiple applications in biological contexts [31, 32]. Until now, there has been a lack of a suitable approach for controlling nonlinear dynamical systems with multilayer networked structures. Determining how to control a nonlinear multilayer networked system still is a crucial and challenging topic.

In this study, we focus on nonlinear multilayer networked systems, which consist of a fixed set of nodes connected by different types of links, and each layer has a complicated nonlinear dynamical process. To explore a general framework for controlling nonlinear multiplayer networked systems, we propose a novel strategy for formulating the problem as a minimum union optimization problem. In particular, we offer a novel approach for identifying the minimum set of driver nodes that steer a multilayered nonlinear dynamical system toward any desired dynamical attractor. Three disease-related biological multilayer networks are used to illustrate the effectiveness of our proposed approach. We discovered that in the minimal set of driver nodes, which were identified using the proposed algorithm, some nodes could act as drug targets in biological experiments. Other nodes were also demonstrated to be involved in important biological processes from the existing literature.

\section{Results}

We investigated the minimal feedback vertex set (MFVS) of three regulatory multilayer networks, including the Colitis-Associated Colon Cancer (CACC) network, Human-HIV1 Multiplex Gpi (HHMG) network and Cancer-Immune Cell-Cell (CICC) interaction network (Figs. 1, 2 and 3, respectively). We demonstrated that our proposed method is capable of determining the MFVS of networks such that the steering nodes in the MFVS can steer a multilayer nonlinear dynamical system. The data sources used in constructing multilayer networks are shown in Table 1. The identified driver nodes for these three networks are listed in Table 2, and their detailed functions are shown in Additional file 1: Table S1.

\section{Colitis-Associated Colon Cancer (CACC) network}

F. Balkwill et al. [33] discovered that inflammation and cancer are closely related. Lu et al. [34] investigated inflammation-associated cancer and constructed a CAC network with 70 nodes and 154 regulatory interactions by exploring the GeneGo database.

To deeply understand the mechanisms of inflammation and cancer, we integrate colon cancer data from different sources, including the DirectedPPI database (www.flyrnai.org/DirectedPPI), KEGG database (www. kegg.jp/) and the work of Lu et al. [34] to build a duplex network (Fig. 1). The data sources are shown in the first column of Table 1.

Applying our method to the CACC network, we identify the two-layer MFVS, which can drive this multilayer networked system from an arbitrary initial state to any desired dynamical attractor by providing proper external signals. There are 17 steering nodes, including AKT, CASP9, P21, BCATENIN, IFNG, IL4, JAK, JUN, NFKB, IKB, PI3K, RAF, SMAD, SPHK1, P53, TREG and IAP in the identified MFVS. Among these nodes, there a 13 steering nodes, including AKT, P21, BCATENIN, IFNG, JAK, JUN, NFKB, 


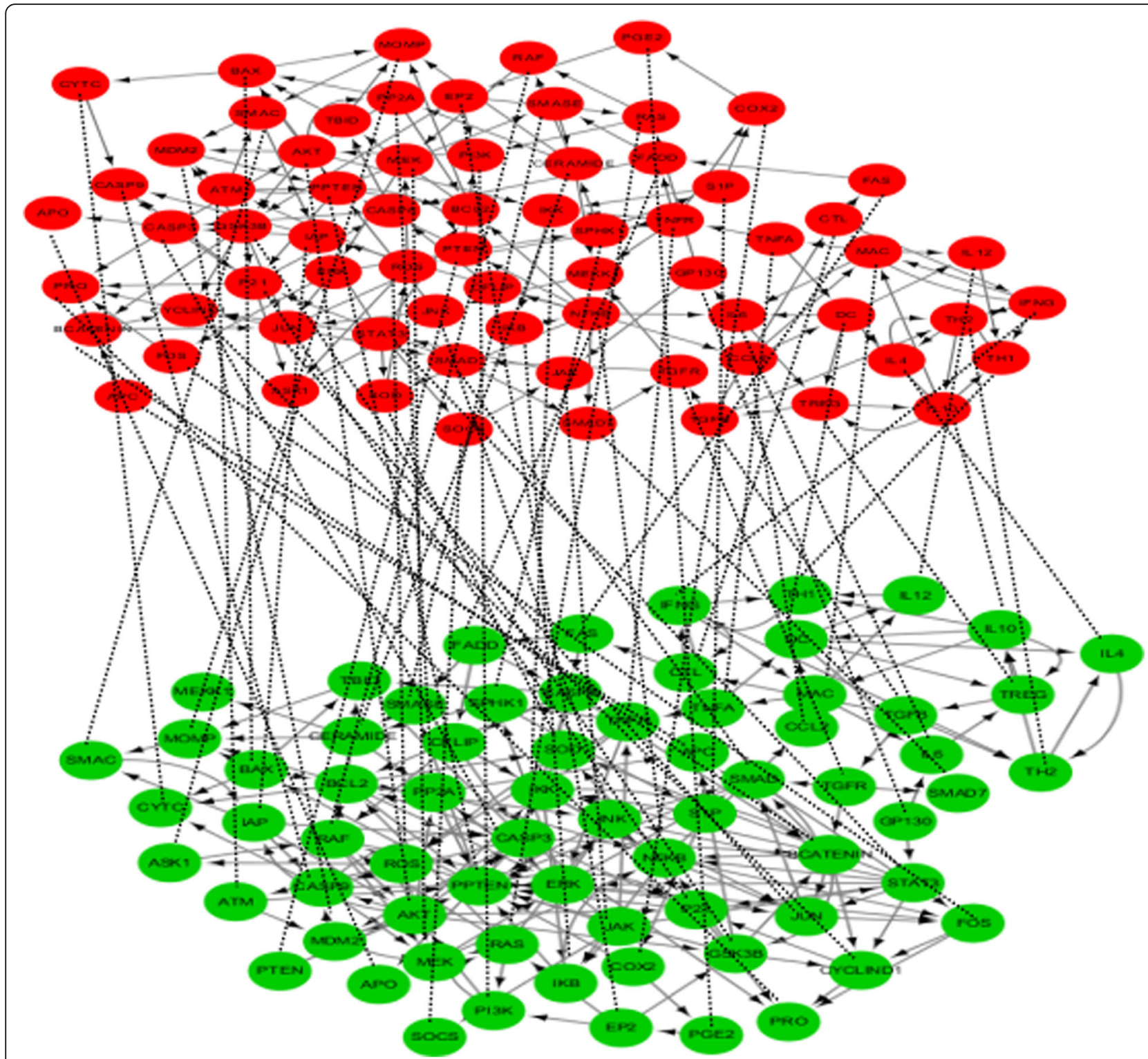

Fig. 1 Colitis-Associated Colon Cancer (CACC) network. The first layer contains 70 nodes and 154 regulatory interactions and the second layer has the same 70 nodes and different 200 different links

IKB, PI3K, RAF, SMAD, P53 and IAP, that are drug targets and each node can interact with 5.00 drugs on average according to the DrugBank database (https:// www.drugbank.ca) [35]. The different names for the drugs are listed in Additional file 1: Table S2.

Though SPHK1, TREG, CASP9 and IL4 cannot be considered as drug targets, they are also involved in important biological processes. SPHK1 plays an important role in tumorigenesis, hormonal therapy, chemotherapy resistance, and it is considered to be a new target for cancer therapeutics [36]. TREG is a type of regulatory $\mathrm{T}$ cell that is one of the major components for immunosuppression and promotes suppressive cytokines as well as inhibiting effector T cells (CD8 T cells and NK cells) directly in the cancer-immune system [37]. Based on the STITCH database (http://stitch.embl.de/) [38], CASP9 can interact with 4 chemicals, namely, cisplatin, 15d-PGJ2, cordycepin and hydrogen peroxide. Of these chemicals, cisplatin is a platinum-based chemotherapy drug used to treat various types of cancers, including sarcomas, some carcinomas (e.g., small cell lung cancer and ovarian cancer), lymphomas and germ cell tumors. IL4 can interact with 4 chemicals, namely, montelukast, ALLERGENS, retinoic acid, and tacrolimus. Tacrolimus is an immunosuppressive drug and retinoic acid is also a medication used for the treatment of some certain 


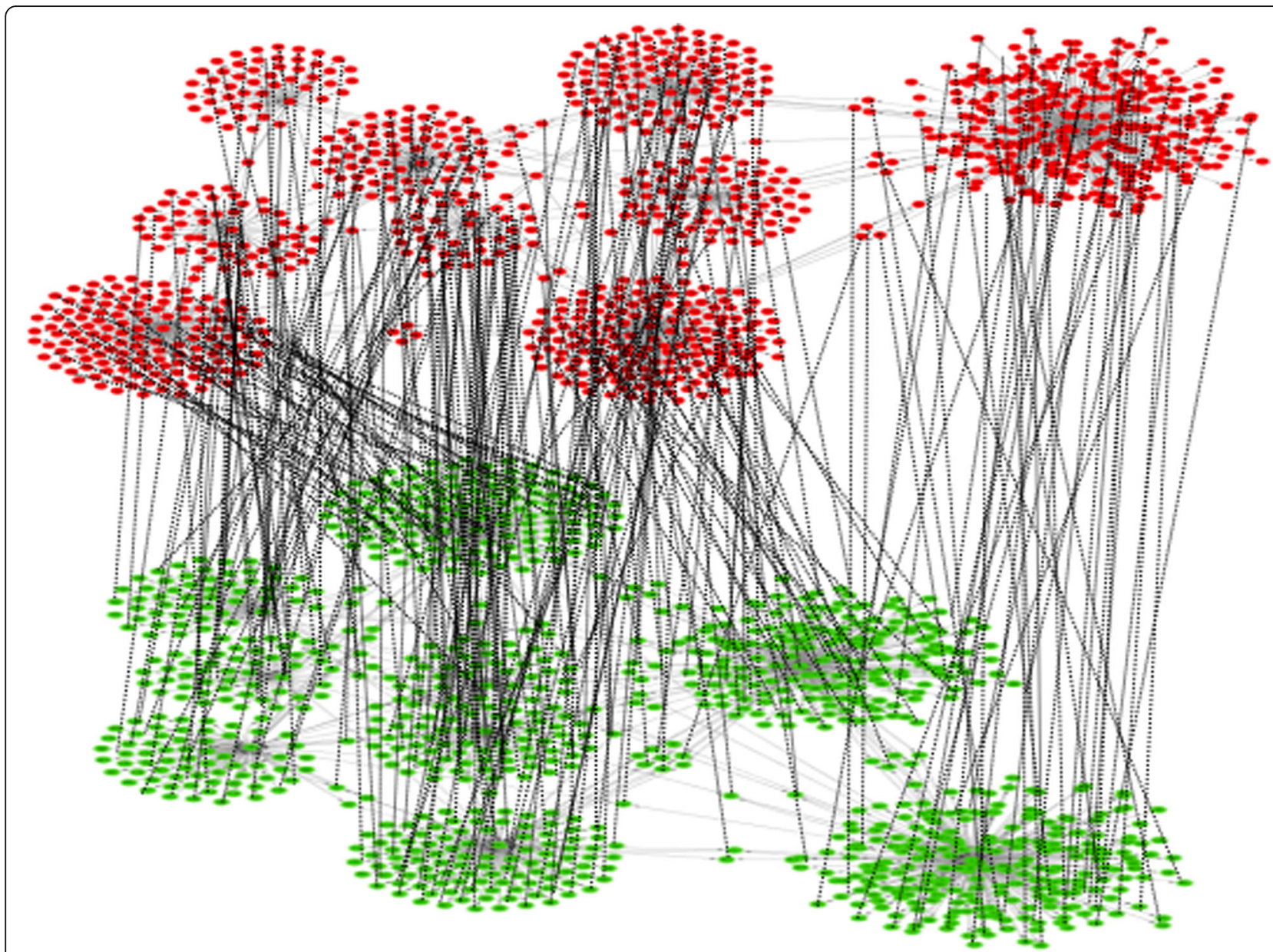

Fig. 2 Human-HIV1 Multiplex Gpi (HHMG) network. The first layer represents physical associations with 1157 edges and 1004 nodes. The second layer represents direct interactions with 1135 edges and 1004 nodes. The interaction relationships are derived from the BioGRID database

cancers. Therefore, we believe SPHK1, TREG, CASP9 and IL4 can be regarded as potential drug targets that are critical for the treatment of colon cancer.

If we only consider a single-layer network, i.e., the CAC network with the SG algorithm, the driver nodes we obtained represented 10 nodes, including AKT, P21, IFNG, IL4, IKB, SMAD, P53, TREG, MEK and STAT3. 7 Proteins AKT, P21, IFNG, IKB, SMAD, P53 and MEK are demonstrated as drug targets from DrugBank database. Moreover, we compared the proportion of drug targets for the different driving nodes (Table 3). Compared to single-layer network (0.7), a multilayer network (0.72\0.77) obtained driver nodes with higher target proportions. These results show that by integrating different interaction relations, multilayer networks can describe biological processes more accurately than single-layer networks, and more significant results can be obtained from them. In particular, the steering nodes with the IISG algorithm are more enriched with known drug targets (0.77), which support the applicability of the method.
Based on DAVID Database (https://david.ncifcrf.gov/ ), the 18 significant pathways of genes in CACC network that $p$ values are less than $7.00 \mathrm{E}-13$ are shown in Fig. 4 and Additional file 1: Figures S1-S5. From Fig. 4 and Additional file 1: Figures S1-S5, we can observe that identified driver nodes are enriched in $\mathrm{T}$ cell and $B$ cell receptor signaling pathways and apoptotic process, which are three of eight hallmarks of cancer. Moreover, we obtained the top 10 genes in pathways through sorting the $\mathrm{p}$ values from small to large by using MSigDB Database (http://software.broadinstitute.org/gsea/msigdb). Additional file 1: Figure S6 illustrates that whether a gene belongs to a particular pathway clearly. Specifically, in "Pathways in cancer" and "Colorectal cancer pathway" with the minimal p values, we discovered the same module of "PI3K-AKT signaling pathway", "MAPK signaling pathway" and "NF- $\mathrm{KB}$ signaling pathway" in these two pathways (Additional file 1: Figures S1-S5), which could provide a novel research direction on colon cancer treatments. 


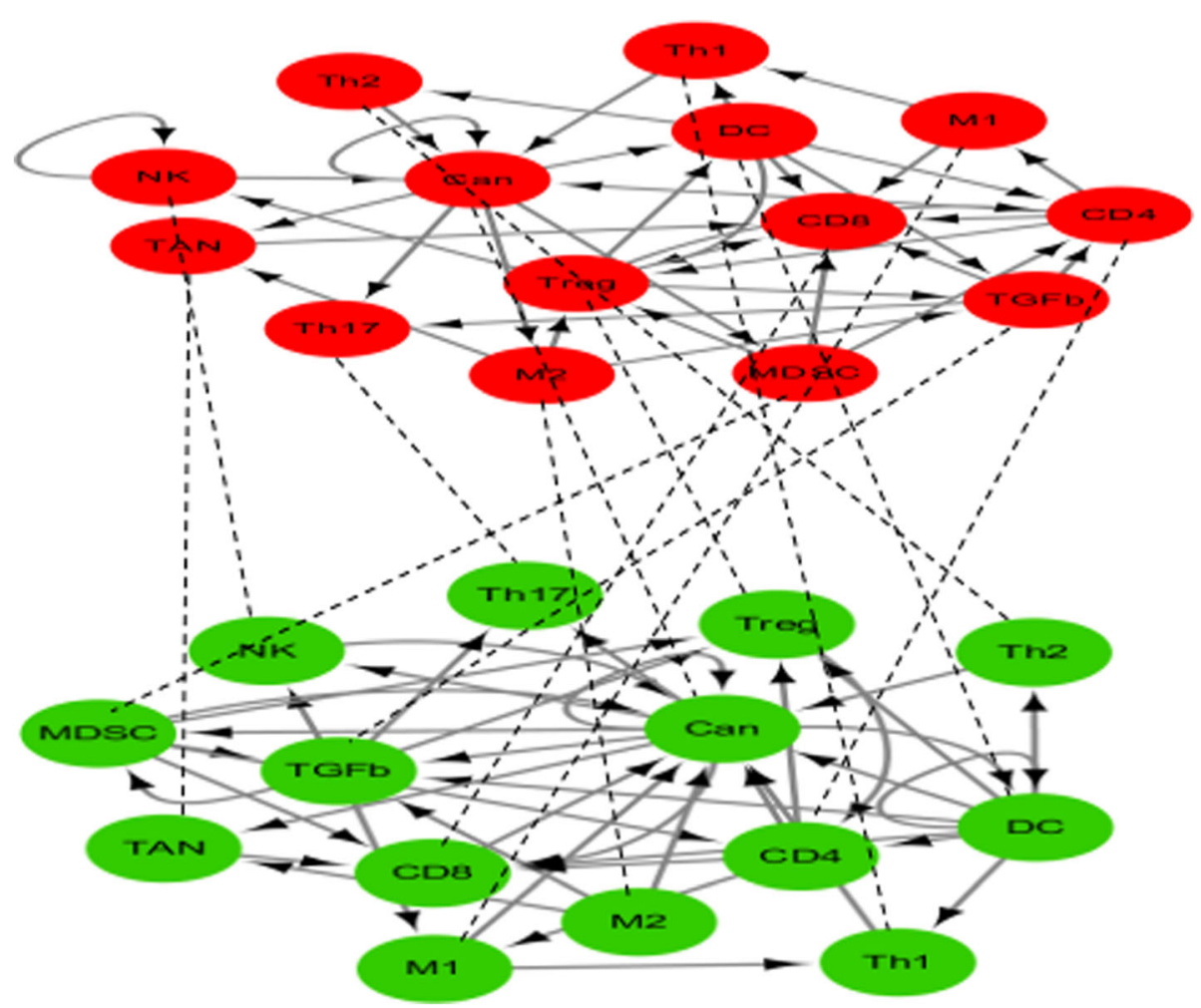

Fig. 3 Cancer-Immune Cell-Cell (CICC) interaction network. The first layer has 14 nodes and 38 links, and the second layer contains the same 14 nodes and 42 regulatory interactions

\section{Human-HIV1 Multiplex Gpi (HHMG) network}

Human Immunodeficiency Virus (HIV) is one of the most notorious viruses that humans have ever faced. Despite many HIV studies conducted over 30 years, the mechanism of HIV remains poorly understood. Researchers found that the infection processes of HIV-1 are related to different types of genetic interactions, which can be represented by a multiplex network (https://comunelab.fbk.eu/data.php). We integrated this HHMG network into a two-layer duplex network (Fig. 2) in which the first layer represents a physical association with 1157 edges and 1004 nodes. The second layer represents direct interaction with 1135 edges and 1004 nodes. The interaction relationships are derived from the BioGRID database and literature.

Applying the IISG algorithm to the HHMG network, we identified the MFVS with 9 steering nodes, including VPR, TAT, ENV, GAG-POL, GAG, NEF, VPU, REV and
VIF, which are shown in the second column of Table 2. More importantly, four proteins, including ENV, GAG-POL, GAG and NEF, were experimentally validated to be drug targets in the DrugBank Database (Additional file 1: Table S1). The different names for the drugs are listed in Additional file 1: Table S3. Moreover, another five nodes, which are VPR, TAT, VPU, REV and VIF, were also involved in important biological processes. HIV-1 encodes VPR, which is a 96-amino-acid protein that can prevent proliferation of infected cells by acting primarily as a cytostatic drug [39]. It has been revealed that the mechanism of TAT activation involves RNA Polymerase II elongation of the integrated HIV-1 [40]. VPU, which is an accessory protein that is encoding by HIV-1, is involved in several immunomodulatory functions, including counteraction of the host restriction factor tetherin and down modulation of CD4 $\mathrm{T}$ cells [41]. In addition to controlled processing of RNA, HIV-1

Table 1 Data sources for three multilayer networks: CACC, HHMG and CICC networks

\begin{tabular}{|c|c|c|c|c|c|}
\hline \multicolumn{2}{|l|}{ CACC network } & \multicolumn{2}{|l|}{ HHMG network } & \multicolumn{2}{|c|}{ CICC network } \\
\hline First layer & Second layer & First layer & Second layer & First layer & $\begin{array}{l}\text { Second } \\
\text { layer }\end{array}$ \\
\hline $\begin{array}{l}\text { CAC network } \\
\text { [34] }\end{array}$ & $\begin{array}{l}\text { DirectedPPI database +KEGG } \\
\text { database+ CAC network [34] }\end{array}$ & $\begin{array}{l}\text { CoMuNe lab } \\
\text { (https://comunelab.fbk.eu/data.php) }\end{array}$ & $\begin{array}{l}\text { CoMuNe lab } \\
\text { (https://comunelab.fbk.eu/data.php) }\end{array}$ & $\begin{array}{l}\mathrm{ClC} \\
\text { network } \\
{[47]}\end{array}$ & $\begin{array}{l}\text { CIS } \\
\text { network } \\
{[48]}\end{array}$ \\
\hline
\end{tabular}


Table 2 Identified driver nodes for three multilayer networks: CACC, HHMG and CICC networks

\begin{tabular}{|c|c|c|c|}
\hline \multicolumn{2}{|c|}{ CACC network (17) } & \multirow{2}{*}{$\begin{array}{l}\text { HHMG network (9) } \\
\text { ENV }\end{array}$} & \multirow{2}{*}{$\begin{array}{l}\text { CICC network (5) } \\
\text { TGFß }\end{array}$} \\
\hline AKT & RAF & & \\
\hline P21 & SMAD & GAG-POL & Cancer cells \\
\hline BCATENIN & P53 & GAG & NK cells \\
\hline IFNG & IAP & $\mathrm{NEF}$ & CD $4 \mathrm{~T}$ cells \\
\hline JAK & CASP9 & VPU & DC \\
\hline JUN & IL4 & REV & \\
\hline NFKB & SPHK1 & VIF & \\
\hline IKB & TREG & VPR & \\
\hline PI3K & & TAT & \\
\hline
\end{tabular}

replication is also dependent on the activities provided by TAT and REV encoding by HIV-1 [40]. VIF, HIV-1 accessory protein is necessary for the production of infectious virions by CD4 lymphocytes [42] Moreover, according to the STITCH Database, VPR, TAT, REV and VIF can interact with 1, 5, 2, 4 kinds of chemicals, respectively. Therefore, VPR, TAT, VPU, REV and VIF can be considered potential drug targets for treating the HIV-1.

To further demonstrate the advantages of using multilayer network, we compared the results using only a single-layer network and multilayer network. Table 4 lists the driver nodes using the first layer network (DNL1), the second layer network (DNL2) and a network with two layers (DNL3). From Table 4, we can observe that the main difference is the obtained protein ENV, which is an important drug target because it can combine with 8 kinds of drugs according to DrugBank (Additional file 1: Table S3). We also analyzed the gene enrichment of genes in MFVS of the HHMG network. From the results in Additional file 1: Table S4, we can see that these genes are involved in host-virus interactions, which are agreement with the experimental evidences.

\section{Cancer-Immune Cell-Cell (CICC) interaction network} Immune cells have been suggested to play paramount roles in controlling malignancy [43, 44]. Recently, large efforts have been devoted to cancer immunotherapy by investigating the cancer-immunity interaction network [45, 46]. Li [47] mapped the complicated interactions among cancer cells and immune cells onto a cancer-immunity

Table 3 Comparisons of the proportion of drug targets using three different methods, including SG, ISG and the proposed IISG for the CAC and CACC networks

\begin{tabular}{ll}
\hline & Proportion of Drug Targets \\
\hline SG algorithm with CAC network & 0.7 \\
ISG algorithm with CACC network & 0.72 \\
IISG algorithm with CACC network & 0.77 \\
\hline
\end{tabular}

cell-cell interaction network (CIC network) including 10 nodes (9 representative cell types and an important cytokine) and 28 interaction links to explore the biological principles between cancer and immunity. Similarly, Wang et al. [48] constructed a comprehensive cancer-immune system (CIS network) including 26 nodes (13 representative cell types and 13cytokine) and 107 interaction links by collecting data from the existing literature.

To deeply understand the mechanisms between the cancer and the immune system, we integrate the CIC network and CIS network to build a duplex network (Fig. 3). The data sources are shown in the third column in Table 1. Applying the IISG algorithm to the CICC network, we identified the MFVS for two layers with five nodes, which was reduced by one ("TREG") compared to the ISG algorithm (see the last row in Table 5). To further demonstrate the advantages of using a multilayer network, we compare the results using only a single-layer network and multilayer network. Table 5 lists the driver nodes using the first layer network (DNL1), the second layer network (DNL2) and a network with two layers (DNL3). From Additional file 1: Table S5, we can see that the identified TGFß is considered to be a critical suppressor of $\mathrm{T}$ cell activities which kill the tumor cells directly [37]. TGFß leads to cancer proliferation [49]. Cancer cells and natural killer (NK) cells have a self-growth system. Cancer cells are the major target we would like to investigate and the NK cells, by boosting the immune system and restricting the growth of tumors, reconsidered to be one of the major inhibitors of tumor cells. Increasing the proliferation of NK cells is an anticancer strategy [47]. By promoting the activity of CD8, CD4 and TREG cells, dendritic cells (DC) provide major mechanisms for $\mathrm{T}$ cell activation. CD4 T cells are one of the inhibiting cell types for cancer. CD4 T cells are an equally critical component for the antitumor immune response. Successful immunity to cancer therefore requires activation of tumor-specific CD4 $\mathrm{T}$ cells [50]. In Additional file 1: Table S5, we display the detailed biological information of cytokines and cell types related to cancer immune system. To sum up, these results demonstrated that in cancer-immune related cell types and cytokines, five nodes including four cell types (Cancer cells, NK cells, DC and CD4 T cells) and one cytokine (TGFß), are critical for discovering the biological principles and insights that clarify the interplay between cancer and immunity, and these nodes are identified targets for cancer immunotherapy.

\section{Discussion}

In this study, we investigated the nonlinear control of multilayer networks by proposing a novel approach, the IISG algorithm, for identifying the minimal set of driver 


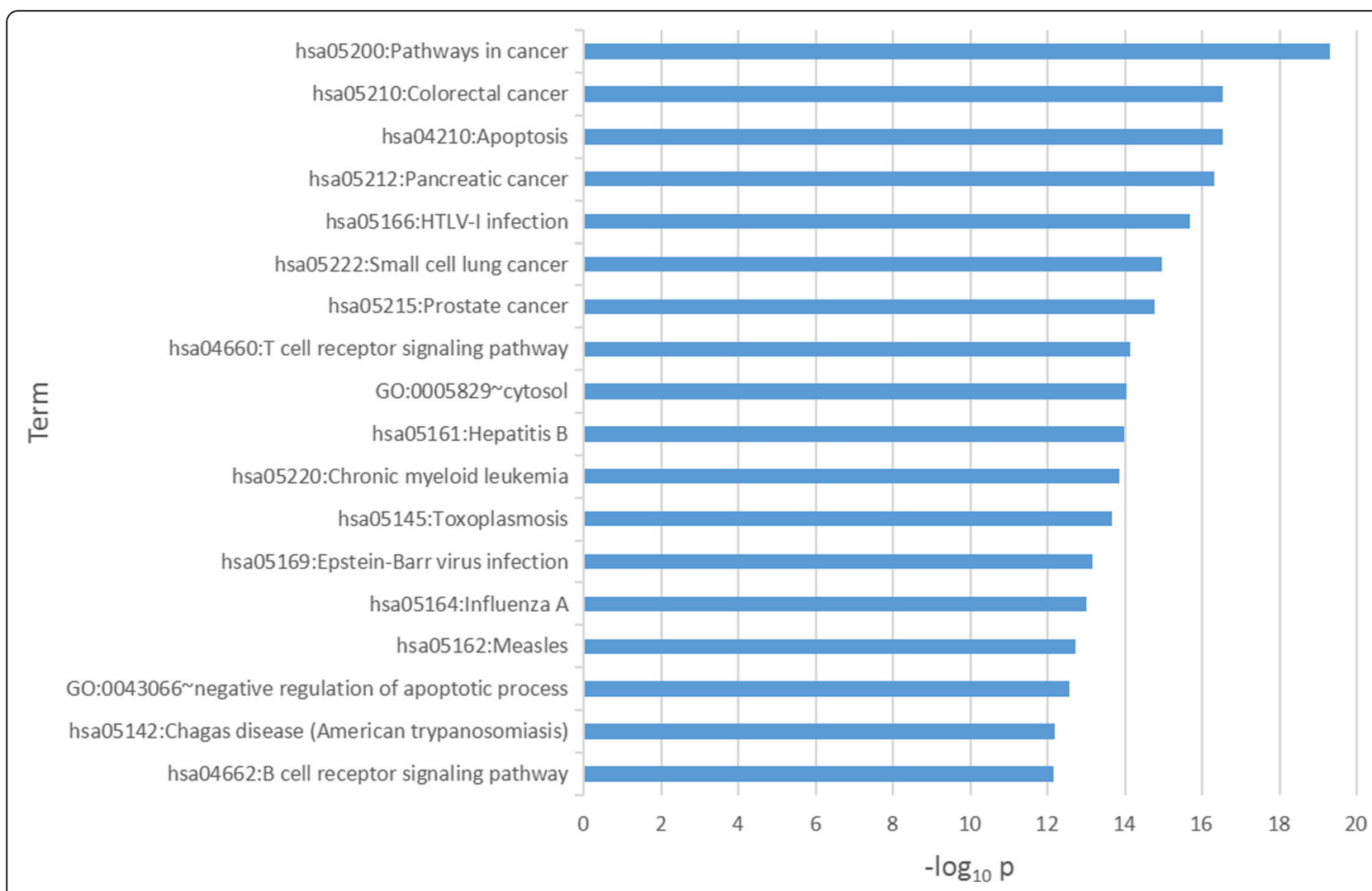

Fig. 4 The 18 significant pathways of genes in CACC network and $p$ values are less than 7.00E-13. The $p$ values of pathways in cancer and Colorectal cancer pathway are minimal

nodes that steer a multilayered nonlinear dynamical system toward any desired dynamical attractor.

We applied the algorithm to three real biological networks, including the CACC, HHMG and CICC networks. The IISG algorithm is capable of obtaining the minimal FVS of a duplex network compared to the ISG algorithm. Through comparisons of the results using a single-layer network and a multilayer network, its effectiveness are demonstrated. In future work, we will extend these approaches to drug targets of complex diseases.

It has been demonstrated that diabetic pathway biomarkers can be identified from gene expression profiling data [51]. Therefore, in future work, we will focus on integrating disease-related signaling networks in the network controllability study and designing methods for

Table 4 Comparisons of identified driver nodes using singlelayer and multilayer networks for the HHMG network

\begin{tabular}{llllllllll}
\hline DNL1 & VPR & TAT & ENV & & GAG- & GAG NEF & VPU & VIF \\
\hline DNL & VPR & TAT & CD 4T & GAG- & GAG & NEF & VPU & REV VIF \\
2 & & cells & POL & & & & \\
DNL & VPR & TAT & ENV & & GAG- & GAG NEF & VPU & REV VIF \\
3 & & & & POL & & & & \\
\hline
\end{tabular}

identifying drug targets and biomarkers for specific genes. In addition, other multilayer nonlinear controllability objectives, such as an improved algorithm for larger multilayer networks, could be explored in future work. In addition, other meaningful node information, such as drugs specificity and particular node functions could be considered for identifying meaningful MFVS for multilayer networks.

\section{Conclusions}

In this study, we have presented a computational framework for detecting the driver nodes in multilayer networks and applied to three real networks. The obtained results suggest that our proposed method could be a promising tool for higher drug target enrichment or discovering more meaningful steering nodes. This work can provide a good foundation for exploring and analyzing complex networks based on big data sets.

Table 5 Comparisons of identified driver nodes using singlelayer and multilayer networks for the CICC network

\begin{tabular}{|c|c|c|c|c|c|}
\hline DNL1 & & Cancer cells & NK cells & & TREG \\
\hline$\overline{\mathrm{DNL} 2}$ & TGFß & Cancer cells & & CD 4 T cells & DC \\
\hline DNL3 & TGFß & Cancer cells & NK cells & CD $4 \mathrm{~T}$ cells & DC \\
\hline
\end{tabular}




\section{Methods}

Mathematical model of nonlinear multilayer networks Most real-world biological systems are multilayer networked structures characterized by different types of interrelationships and extremely complicated nonlinear processes. In this study, we focus on nonlinear multiplayer networked systems, which consist of a fixed set of nodes connected by different types of links (Fig. 5) and each layer is a complicated nonlinear dynamical process. To explore the controllability of multilayer networks with nonlinear dynamics, a system with $S$ layers and $N$ nodes can be described with the following ordinary differential equations (ODEs):

$$
x(t) \cdot=F(x)+B u(t)
$$

Where $x(t)=\left[x^{(1)}, x^{(2)}, \cdots, x^{(S)}\right]^{T}$ and $x^{(\alpha)}=\left(x_{1}^{(\alpha)}, x_{2}^{(\alpha)}, \cdots, x_{N}^{(\alpha)}\right)^{T}$ $(\alpha=1,2, \cdots, S)$ represent the state of nodes in layer $\alpha$. $F(x)=\left[\begin{array}{llll}F^{(1)}(x) & \ldots & F^{(S)}(x)\end{array}\right]^{T}$ and $F^{(\alpha)}=\left(F_{1}^{(\alpha)}, F_{2}^{(\alpha)}, \cdots, F_{N}^{(\alpha)}\right)^{T}$ $(\alpha=1,2, \cdots, S)$ is a continuously nonlinear differentiable vector function for layer $\alpha . B=\operatorname{diag}\left(B^{(1)}, B^{(2)}, \cdots, B^{(S)}\right)$ $\in \mathbb{R}^{N S \times M}$ is the control matrix and $B^{(\beta)}(\beta=1,2, \cdots, S)$ are the $N \times P^{\beta}$ matrices describing the coupling between the nodes of each layer $\alpha$ and $P^{\beta} \leq N$ external signals.
The controllability of nonlinear multilayer networks was studied under the assumption that driver nodes are the same in all layers, which mimics the situation in which input nodes can send different signals in the different layers of the multiplex but the position of the external signals in the layers is correlated. In this study, the objective of control problems for multilayer networks with nonlinear dynamics is to identify the minimal driver nodes in the system (1) that steer this multilayered nonlinear dynamical system toward any desired dynamical attractor of each layer.

\section{The minimum union optimization model for controlling} nonlinear multilayer networks

In this section, we present a minimum union optimization model for identifying the minimum set of driver nodes that steer the system (1) toward any desired dynamical attractor of each layer.

In the nonlinear single-layer networks, Fiedleret al. [14] showed that controllability of a single network can be mapped to the feedback vertex set problem. The explanation of FVS is shown in Fig. 6. In real multilayer networks, however, nodes are usually univocally defined and share common properties across different layers, therefore, we made the assumption that each node of the multilayer network is either a driver node in each layer or it is not a driver node in any layer.

Based on the above assumption, the problem of finding the minimal set of driver nodes in the nonlinear multilayer

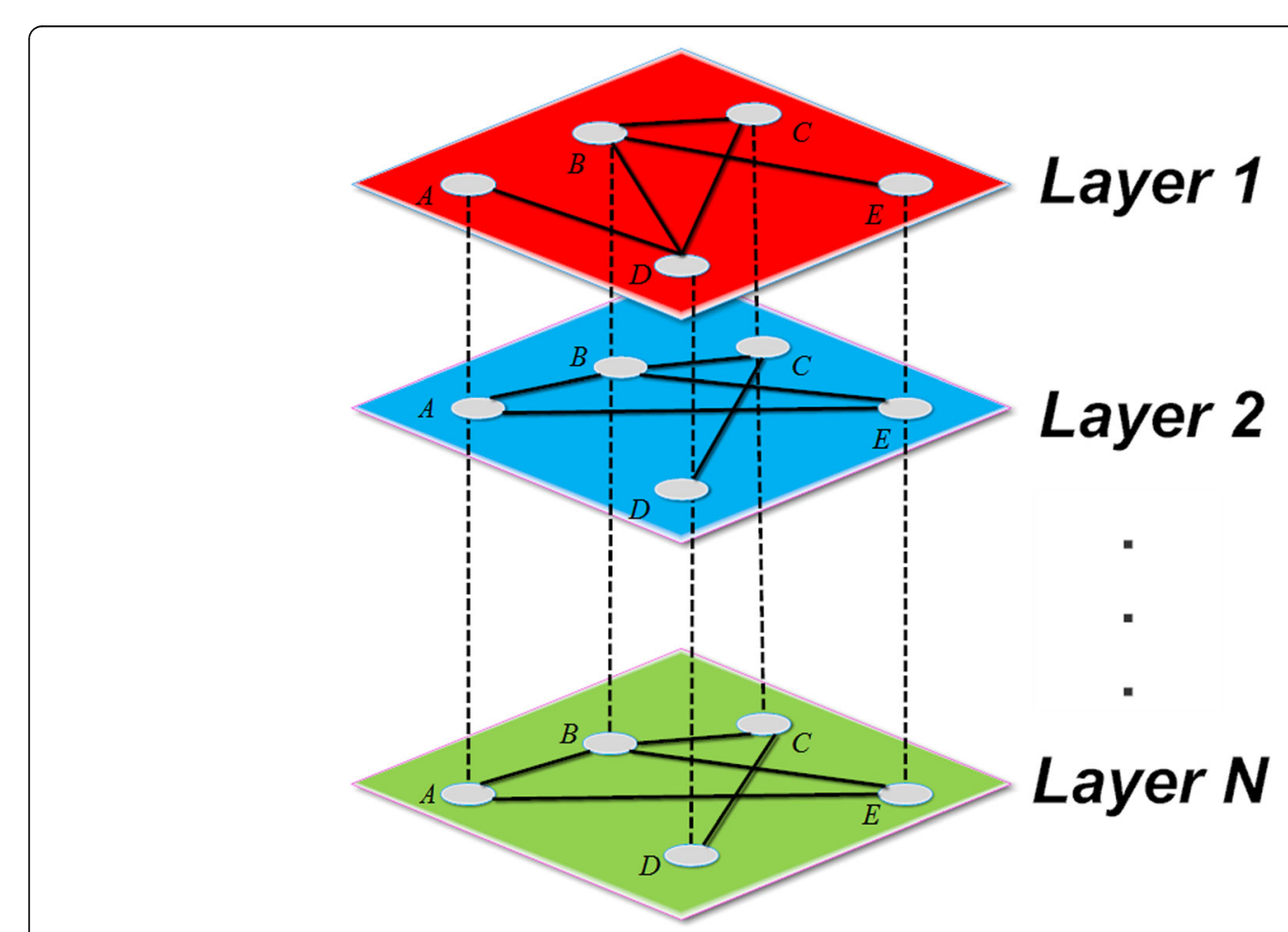

Fig. 5 The $\mathrm{N}$-layer network shares the same set of nodes and different interactions 


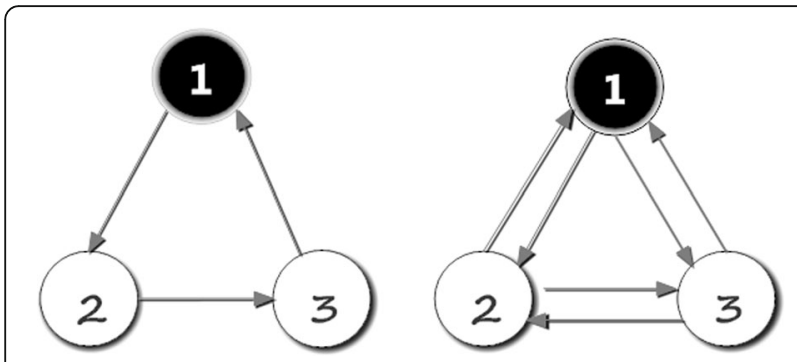

Fig. 6 Schematic illustration of FVS in single-layer networks. The black vertices are one choice for a minimal feedback vertex set in two single-layer networks. The feedback vertices set is a subset of vertices in a directed graph, such that the removal of the set leaves the graph without directed cycles

network can be thus formulated as a minimum FVS union optimization problem, which can drive the nonlinear multilayer networked system from an arbitrary initial state to any desired dynamical attractor. Mathematically, we formulate the nonlinear control of multilayer networks as a minimum FVS union optimization problem as follows:

$$
\min F_{1} \cup F_{2} \cup \ldots \cup F_{n}
$$

Where $F_{i}$ is the number of FVS in layer $i$, which is a set of driver nodes of the layer $i(i=1,2, \ldots, N)$. The model can be illustrated as shown in Fig. 7.

\section{The algorithm for solving the minimum union optimization model}

Currently, several algorithms have been applied to investigate the MFVS in a single-layer network. Razgon [52] proposed the GetMAS algorithm to find the MFVS of a directed graph in $O\left(2^{n}\right)$. To save time, Cai X. [53] investigated simplification of a directed graph when calculating the MFVS. We combined the two concepts and proposed the simplified GetMAS (SG) algorithm to quickly obtain the MFVS of single-layer networks. Thus, the MFVS of a multilayer network can be solved as follows:

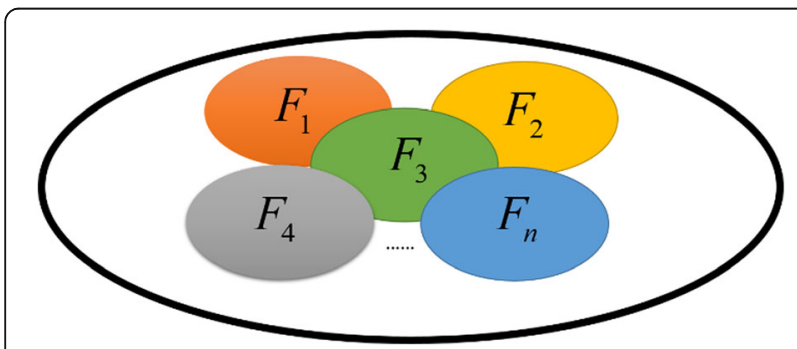

Fig. 7 Illustration of the minimum FVS union optimization problem in a multilayer network. Suppose that $F_{1}, F_{2}, \ldots, F_{N}$ is the FVS in layer 1 , layer $2, \ldots$, layer $N$ respectively. The problem is converted to find the minimal union of $F_{1}, F_{2}, \ldots, F_{N}$, namely, $\min F_{1} \cup F_{2} \cup \ldots \cup F_{N}$
1)Find the feedback vertex set of each layer with the $\mathrm{SG}$ algorithm.

2)Get an intersection of the above sets.

We named the method of getting the intersection of FVS directly as the intersection simplification GetMAS (ISG) algorithm. However, the feedback vertex set from the ISG algorithm is not a minimal set of driver nodes. The algorithm presented in this study was meant to optimize the ISG algorithm.

The improved ISG (IISG) algorithm based on a greedy principle was proposed to identify the MFVS of multilayer networks with $N(N \geq 2)$ layers. Before introducing the algorithm, we define three following concepts.

Definition 1:(Reference layer): The reference layer is the layer for which we first obtain the FVS with the SG algorithm.

Definition 2:(Study layer): The study layer is the layer for which we obtain the FVS with the IISG algorithm. Definition 3:(Dominated nodes): Dominated nodes consists of three kinds of nodes: nodes with more loops, nodes in the FVS of the reference layer and nodes with more functions.

Therefore, the IISG algorithm for finding the MFVS can be described in the following steps:

Step 1: In the reference layer, the set $F_{r}$ (the set of reference layer FVS nodes) is obtained with the SG algorithm.

Step 2: In the study layer, calculate $L_{i}(i=2,3, \ldots, N)$ (the set of the passing loops of all nodes in set $F_{r}$ ).

Step 3: In the study layer, choose the dominated nodes and remove them from the study layer to set $F_{i}(i=2,3$, $\ldots, N$ ) (which is the set of study layer FVS nodes). Calculate $L_{i}(i=2,3, \ldots, N)$.

Step 4: Judge whether the set $L_{i}(i=2,3, \ldots, N)$ is empty or not. If it is an empty set, execute step 5; otherwise, repeat Steps 2 and 3.

Step 5: In the study layer, calculate $L_{i}{ }^{\prime}(i=2,3, \ldots, N)$ (the collection of all the passing loops of the current nodes in the study layer after removing dominated nodes from the study layer to set $\left.F_{i}(i=2,3, \ldots, N)\right)$. Step 6: In the study layer, choose the dominated node sand remove them from the study layer to set $F_{i}(i=2,3, \ldots$,

$N)$. Calculate $L_{i}^{\prime}(i=2,3, \ldots, N)$.

Step 7: Judge whether the set $L_{i}^{\prime}(i=2,3, \ldots, N)$ is empty or not. If it is an empty set, execute step 8; otherwise, repeat Steps 5 and 6.

Step 8: $F_{1,2, \ldots, \text { i }}$ the minimal driver nodes of a multilayer network where there is a multiplex with $N=i$ layer $)$ is the union of $F_{r}$ and $F_{i}(i=2,3, \ldots, N)$. 
In particular, when the number of layers in a multilayer network exceeds two, the set $F_{1,2, \ldots, \text { i }}$ is regarded as the set $F_{n}$ and then we can start the next calculation.

The pseudocode of the IISG algorithm is presented as follows.

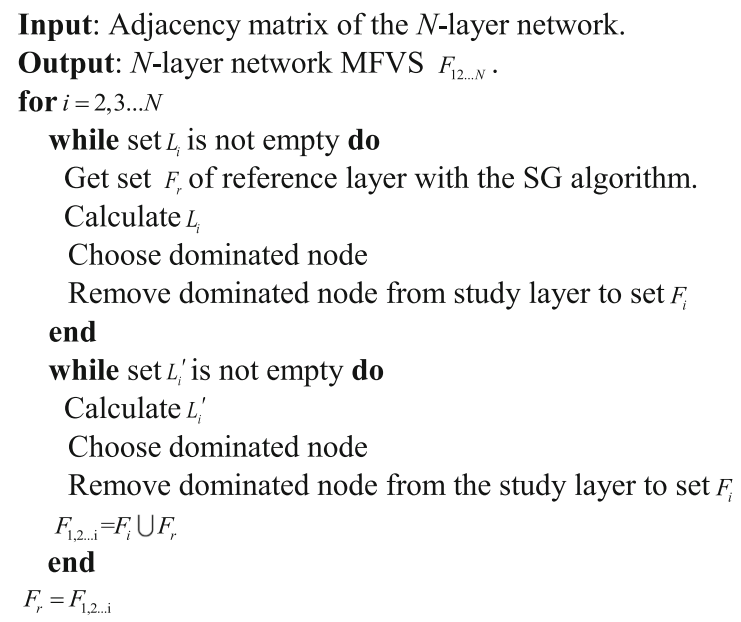

End

Where $F_{r}$ is the set of reference layer FVS nodes with the SG algorithm and $L_{i}(i=2,3, \ldots, N)$ is the set of the passing loops of all nodes in set $F_{r}$ in the study layer. $F_{i}(i=2,3, \ldots, N)$ is the set of study layer FVS nodes. $L_{i}{ }^{\prime}(i=2,3, \ldots, N)$ is the collection of all the passing loops of the current nodes in the study layer after removing the dominated nodes from the study layer to set $F_{i}$. Set $F_{12, \ldots, \mathrm{n}}$ is the minimal driver nodes of the $N$-layer network.

To further illustrate the IISG algorithm, we consider a simple duplex network in Fig. 8. Because there is no biological process involved in this example, dominated nodes with more biological functions were not considered when the dominated nodes were selected. The nodes numbered 2 and 5 are one choice for MFVS in the reference layer (the layer with red nodes in Fig. 8). The 4 circles, 2, 2; 1 , 2,$5 ; 2,5,4,3$ and $1,5,7$, were found in the study layer (the layer with green nodes in Fig. 8). Nodes 2 and 5 pass through the most circles ( 3 circles) 2, 2; $1,2,5 ; 2,5,4,3$ and $1,2,5 ; 2,5,4,3 ; 1,5,7$, respectively, and there are also nodes in the reference layer FVS. Therefore, we consider nodes 2 and 5 as the dominated nodes and as members of FVS in the study layer. Next, they are removed from the study layer. In the study layer, there were only nodes 1, 3, 4, 6 and 7, which contained only one circle for 3 and 4 . If it is a specific biological problem, we can choose a node as the FVS according to the biological function of nodes 3 and 4 . In this study, we temporarily selected node 3 . In conclusion, the FVS of the reference layer contains nodes 2 and 5 and the FVS of the study layer contains nodes 2, 5 and 3. Therefore, the MFVS of the duplex network contains nodes 2,5 and 3 .

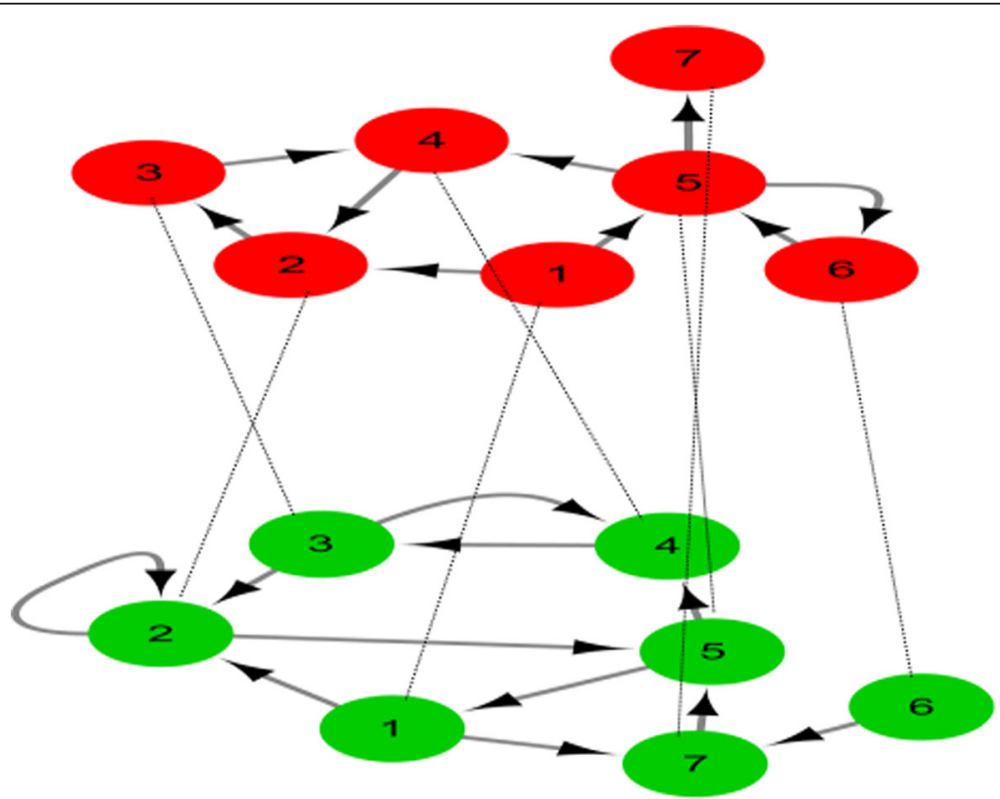

Fig. 8 Control of a duplex network (multiplex with $N=2$ layers). The layer with red nodes is the reference layer and the layer with green nodes is the study layer 


\section{Additional file}

Additional file 1: Figure S1. In Pathways in cancer, the above genes are clustered near the PI3K-AKT signaling pathway. Figure S2. In Colorectal cancer pathway, the above genes are clustered near the PI3KAKT signaling pathway and MAPK signaling pathway. Figure S3. In Apoptosis pathway, the above genes are clustered near the PI3K-AKT signaling pathway and NF-KB signaling pathway. Figure S4. In B cell receptor signaling pathway, the above genes are clustered near the PI3K-AKT signaling pathway, MAPK signaling pathway and NF-KB signaling pathway. Figure S5. In T cell receptor signaling pathway, the above genes are clustered near the MAPK signaling pathway and NF-KB signaling pathway. Figure S6. The enrichment analysis of genes from MsigDB database. Table S1. Biological functions of nodes in MFVS for three multilayer networks. Table S2. The names of drugs that AKT, P21, BCATENIN, IFNG, JAK, JUN, NFKB, IKB, PI3K, RAF, SMAD, P53 and IAP can combine with. Table S3. The drugs which ENV, GAG, NEF and GAG-POL can combine with. Table S4. Enrichment analysis of genes in MFVS of the HHMG network. Table S5. The biological information of cytokines and cell types related to cancer immune system. (PDF $626 \mathrm{~kb}$ )

\section{Acknowledgements}

The authors thank Prof. FangXiang Wu in University of Saskatchewan,CA for providing valuable suggestions on the paper.

\section{Funding}

This work was supported by the National Key Research and Development Program of China (No. 2018YFC1314600) and the Chinese National Natural Science Foundation (No. 11831015 and No. 61672388). The funding body did not play any roles in the design of the study and collection, analysis, and interpretation of data and in writing the manuscript.

\section{Availability of data and materials}

The datasets used and/or analyzed during the current study are available from the corresponding author on reasonable request.

\section{Authors' contributions}

XFZ designed the study, WZ proposed the algorithm and realized the application of the algorithm. DW collected data information. WZ and XFZ wrote and revised the manuscript. All authors read and approved the final manuscript.

\section{Ethics approval and consent to participate}

Not applicable.

\section{Consent for publication}

Not applicable.

\section{Competing interests}

The authors declare that they have no competing interests.

\section{Publisher's Note}

Springer Nature remains neutral with regard to jurisdictional claims in published maps and institutional affiliations.

Received: 1 April 2019 Accepted: 22 April 2019

Published online: 28 May 2019

\section{References}

1. Ruths J, Ruths D. Control profiles of complex networks. Science. 2014; 343(6177):1373-1376.

2. Liu YY, Slotine JJ, Barabási AL. Controllability of complex networks. Nature. 2011;473(7346):167-173.

3. Wang D, Jin S, Wu F-X, Zou X. Estimation of control energy and control strategies for complex networks. Adv Complex Syst. 2015; 18(07n08):1550018.

4. Yan G, Vértes PE, Towlson EK, Chew YL, Walker DS, Schafer WR, Barabási A-L. Network control principles predict neuron function in the Caenorhabditis elegans connectome. Nature. 2017;550(7677):519-523.
5. Yuan Z, Zhao C, Di Z, Wang W-X, Lai Y-C. Exact controllability of complex networks. Nat Commun. 2013:4:2447.

6. Mochizuki A, Fiedler B, Kurosawa G, Saito D. Dynamics and control at feedback vertex sets. II: A faithful monitor to determine the diversity of molecular activities in regulatory networks. Journal of Theoretical Biology. 2013;335:130-146

7. Cornelius SP, Kath WL, Motter AE. Realistic control of network dynamics. Nat Commun. 2013:4:1942

8. Jgt ZU, Yang G, Albert R. Structure-based control of complex networks with nonlinear dynamics. Proc Natl Acad Sci. 2017;114(28):7234-7239.

9. Wang L-Z, Su R-Q, Huang Z-G, Wang X, Wang W-X, Grebogi C, Lai Y-C. A geometrical approach to control and controllability of nonlinear dynamical networks. Nat Commun. 2016:7:11323.

10. Liu YY, Slotine JJ, Barabási AL. Observability of complex systems. Proc Natl Acad Sci. 2013:110(7):2460-2465.

11. Yan G, Ren J, Lai Y-C, Lai C-H, Li B. Controlling complex networks: how much energy is needed? Phys Rev Lett. 2012:108(21):218703.

12. Yan G, Tsekenis G, Barzel B, Slotine JJ, Liu YY, Barabási A-L. Spectrum of controlling and observing complex networks. Nat Phys. 2015;11(9):779-786.

13. Gao J, Liu Y-Y, D'souza RM, Barabási A-L. Target control of complex networks. Nat Commun. 2014;5:5415.

14. Fiedler B, Mochizuki A, Kurosawa G, Saito D. Dynamics and control at feedback vertex sets. I: Informative and determining nodes in regulatory networks. Journal of Dynamics and Differential Equations. 2013;25(3):563604.

15. Jin S, Wu F-X, Zou X. Domain control of nonlinear networked systems and application to complex disease networks. Discrete \& Continuous Dynamical Systems-B. 2017;22(6):2169-2206.

16. Jin S, Wang D, Zou X. Trajectory control in nonlinear networked systems and its applications to complex biological systems. SIAM J Appl Math. 2018; 78(1):629-649.

17. Klosik DF, Grimbs A, Bornholdt S, Hütt MT. The interdependent network of gene regulation and metabolism is robust where it needs to be. Nat Commun. 2017:8(1):534

18. De Domenico M, Porter MA, Arenas A. MuxViz: a tool for multilayer analysis and visualization of networks. Journal of Complex Networks. 2015;3(2):159176.

19. Boccaletti S, Bianconi G, Criado R, Genio CID, Gómez-Gardeñes J, Romance M, Sendiña-Nadal I, Wang Z, Zanin M. The structure and dynamics of multilayer networks. Phys Rep. 2014;544(1):1-122.

20. Zhen W, Lin W, Szolnoki A, Perc M. Evolutionary games on multilayer networks: a colloquium. Eur Phys J B. 2015;88(5):124

21. De Domenico M, Solè-Ribalta A, Cozzo E, Kivelä M, Moreno Y, Porter MA Gòmez S, Arenas A. Mathematical formulation of multi-layer networks. Physical Review X. 2013:3(4):041022

22. Kivelä M, Arenas A, Barthelemy M, Gleeson JP, Moreno Y, Porter MA Multilayer networks. Journal of Complex Networks. 2014;2(3):203-271.

23. Kenett DY, Perc M, Boccaletti S. Networks of networks - an introduction. Chaos Solitons \& Fractals. 2015:80:1-6.

24. Wang D, Jin S, Zou X. Crosstalk between pathways enhances the controllability of signalling networks. IET Systems Biology. 2016:10(1):2-9.

25. Menichetti G, Dall'Asta L, Bianconi G. Control of multilayer networks. Sci Rep. 2016;6(1):20706.

26. Pósfai M, Gao J, Cornelius SP, Barabási AL, D'Souza RM. Controllability of multiplex, multi-time-scale networks. Phys Rev E. 2016:94(3):032316.

27. Nie S, Wang X, Wang B. Effect of degree correlation on exact controllability of multiplex networks. Physica A: Statistical Mechanics \& Its Applications. 2015;436:98-102.

28. Wang D, Zou X. Control energy and controllability of multilayer networks. Adv Complex Syst. 2017;20(04n05):1750008.

29. Jin S, Li Y, Pan R, Zou X. Characterizing and controlling the inflammatory network during influenza a virus infection. Sci Rep. 2014:4:3799.

30. Li Y, Jin S, Lei L, Pan Z, Zou X. Deciphering deterioration mechanisms of complex diseases based on the construction of dynamic networks and systems analysis. Sci Rep. 2015;5:9283.

31. Liu YY, Barabási AL. Control principles of complex systems. Rev mod phys. 2016;88(3):035006

32. Li A, Cornelius SP, Liu Y-Y, Wang L, Barabási A-L. The fundamental advantages of temporal networks. Science. 2017;358(6366):1042-1046.

33. Balkwill F, Mantovani A. Inflammation and cancer: back to Virchow? The Lancet. 2001;357(9255):539-545. 
34. Lu J, Zeng H, Liang Z, Chen L, Zhang L, Zhang H, Liu H, Jiang H, Shen B, Huang M. Network modelling reveals the mechanism underlying colitisassociated colon cancer and identifies novel combinatorial anti-cancer targets. Sci Rep. 2015:5:14739.

35. Wishart DS, Knox C, Guo AC, Cheng D, Shrivastava S, Dan T, Gautam B, Hassanali M. DrugBank: a knowledgebase for drugs, drug actions and drug targets. Nucleic Acids Res. 2008;36(1):901-906.

36. Dai S, Takabe K, Kapitonov D, Milstien S, Spiegel S. Targeting SphK1 as a new strategy against Cancer. Curr Drug Targets. 2008;9(8):662-673.

37. Robertson-Tessi M, El-Kareh A, Goriely A. A mathematical model of tumorimmune interactions. Journal of Theoretical Biology. 2012;294(2):56-73.

38. Kuhn M, Von MC, Campillos M, Jensen L, Bork P. STITCH: interaction networks of chemicals and proteins. Nucleic Acids Res. 2008;36(1):684-688.

39. Bartz SR, Rogel ME, Emerman M. Human immunodeficiency virus type 1 cell cycle control: Vpr is cytostatic and mediates G2 accumulation by a mechanism which differs from DNA damage checkpoint control. Journal of Virology. 1996;70(4):2324-2331.

40. Balachandran A, Wong R, Stoilov P, Pan S, Blencowe B, Cheung P, Harrigan PR, Cochrane A. Identification of small molecule modulators of HIV-1 Tat and Rev protein accumulation. Retrovirology. 2017;14(1):7.

41. Yamada E, Nakaoka S, Klein L, Reith E, Langer S, Hopfensperger K, Iwami S, Schreiber G, Kirchhoff F, Koyanagi Y. Human-specific adaptations in Vpu conferring anti-tetherin activity are critical for efficient early HIV-1 replication InVivo. Cell Host Microbe. 2018;23(1):110-120

42. Mariani R, Chen D, Schröfelbauer B, Navarro F, König R, Bollman B, Münk C, Nymark-Mcmahon H, Landau NR. Species-specific exclusion of APOBEC3G from HIV-1 Virions by Vif. Cell. 2003;114(1):21-31.

43. Shankaran V, Ikeda H, Bruce AT, White JM, Swanson PE, Old LJ, Schreiber RD. IFNgamma and lymphocytes prevent primary tumour development and shape tumour immunogenicity. Nature. 2001;410(6832):1107-1111.

44. Blattman JN, Greenberg PD. Cancer immunotherapy: a treatment for the masses. Science. 2004;305(5681):200-205.

45. Galluzzi L, Vacchelli E, Bravo-San Pedro JM, et al. Classification of current anticancer immunotherapies. Oncotarget. 2014;5(24):12472-12508.

46. Couzinfrankel J. Breakthrough of the year 2013. Cancer immunotherapy. Science. 2013;342(6165):1432-1433.

47. Li C. Identifying the optimal anticancer targets from the landscape of a cancer-immunity interaction network. Phys Chem Chem Phys. 2017;19(18): 7642-7651.

48. Wenbo L, Wang J. Uncovering the underlying mechanism of cancer tumorigenesis and development under an immune microenvironment from global quantification of the landscape. Journal of The Royal Society Interface. 2017;14(131):20170105.

49. Khaled YS, Ammori BJ, Elkord E. Myeloid-derived suppressor cells in cancer: recent progress and prospects. Immunol Cell Biol. 2013;91(8):493-502.

50. Pardoll DM, Topalian SL. The role of CD4 + T cell responses in antitumor immunity. Curr Opin Immunol. 1998;10(5):588-594.

51. Liu Z-P, Gao R. Detecting pathway biomarkers of diabetic progression with differential entropy. Journal of Biomedical Informatics. 2018;82:143-53.

52. Razgon I. Computing minimum directed feedback vertex set in $\mathrm{O}^{*}(1.9977$ n). Theoretical Computer Science. 2007;10:70-81.

53. Cai $X$, Huang J, Jian $G$. Search algorithm for computing minimum feedback vertex set of a directed graph. Comput Eng. 2006;32(4):67-69.

Ready to submit your research? Choose BMC and benefit from:

- fast, convenient online submission

- thorough peer review by experienced researchers in your field

- rapid publication on acceptance

- support for research data, including large and complex data types

- gold Open Access which fosters wider collaboration and increased citations

- maximum visibility for your research: over $100 \mathrm{M}$ website views per year

At BMC, research is always in progress.

Learn more biomedcentral.com/submissions 